\title{
Myocardial Infarction Caused by Aspergillus Embolization in a Patient with Aplastic Anemia
}

\author{
Mitsuru Itoh ${ }^{4}$, Masahiko Takahashi ${ }^{1}$, Miyuki Mori ${ }^{2}$, Hiromichi Tamekiyo ${ }^{2}$, Hiroshi Yoshida ${ }^{2}$, \\ Kazuhiro Yago ${ }^{1}$, Hideto Shimada ${ }^{1}$ and Kazumori Arai ${ }^{3}$
}

\begin{abstract}
A 38-year-old Japanese man with severe aplastic anemia had invasive pulmonary aspergillosis as a complication. He was treated with amphotericin B for six weeks, but the aspergillosis did not improve. Then he experienced a fatal myocardial infarction. An autopsy revealed disseminated aspergillosis involving pericarditis and Aspergillus embolization to the coronary arteries. This led to the acute myocardial infarction. Cardiac aspergillosis is rare, but should be included within the differential diagnosis when chest pain of unknown origin occurs in an immunosuppressed patient.
\end{abstract}

Key words: aplastic anemia, pericarditis, myocarditis, myocardial infarction, Aspergillus

(DOI: 10.2169/internalmedicine.45.1607)

\section{Introduction}

Disseminated aspergillosis often occurs in immunosuppressed patients and has increased in frequency in recent years. Cardiac involvement of aspergillosis, including myocardial infarction, pericarditis and myocarditis, is comparatively rare. Myocardial infarction secondary to Aspergillus embolization to the coronary arteries has been reported in several patients (1-6), and Aspergillus pericarditis and myocarditis have also been described in some papers (7-17). In this report, we describe a case in an aplastic anemia patient with fungal occlusion of the coronary arteries and fungal invasion of the pericardium and myocardium, although he had been receiving treatment with amphotericin $\mathrm{B}$.

\section{Case Report}

A 38-year-old Japanese man was diagnosed with severe aplastic anemia and hospitalized on February 28, 2000. His bone marrow was characterized by severe hypoplasia. He had undergone a left nephrectomy during childhood. Hematologic findings at diagnosis were white blood cells (WBC)
$0.8 \times 10 \%$ with $0 \%$ neutrophils, $95 \%$ lymphocytes, and $5 \%$ monocytes; platelets were $49 \times 10^{9} / 1$ and hemoglobin $11.6 \mathrm{~g} /$ dl. The bone marrow was severely hypocellular with $78.4 \%$ lymphocytes, and $14.4 \%$ plasmacytes. He was treated with cyclosporin A, granulocyte colony-stimulating factor, corticosteroids and antithymocyte globulin, but they had no effect. The peripheral neutrophil count was zero or less than $0.1 \times 10^{9} / 1$ throughout his clinical course. Erythrocyte and platelet transfusions were frequently necessary. He did not have an HLA-matched sibling. He had pyrexia and cough during mid-March 2000. Serum C-reactive protein was at an aberrantly high level (more than $20 \mathrm{mg} / \mathrm{dl}$ ). His chest X-ray showed pulmonary infiltration of the left lower lobe. Despite treatment with broad-spectrum antibiotics, pneumonia worsened. On March 27, 2000, amphotericin B (0.5 mg/kg/day) was started intravenously because a test for serum Aspergillus antigen was positive and beta-D glucan had increased from $<11$ to $18.7 \mathrm{pg} / \mathrm{ml}$, but his pneumonia did not improve. A fungus ball was visible in the left lower lobe on a chest X-ray. On April 18, 2000, the dosage of amphotericin B was increased $(0.7 \mathrm{mg} / \mathrm{kg} /$ day $)$, but his pneumonia worsened.

On May 5, 2000, he suddenly complained of anterior chest pain. The pain was somewhat relieved by sublingual

\footnotetext{
${ }^{1}$ Division of Hematology and Oncology, Department of Internal Medicine, Shizuoka General Hospital, Shizuoka, ${ }^{2}$ Division of Cardiology, Department of Internal Medicine, Shizuoka General Hospital, Shizuoka, ${ }^{3}$ Department of Pathology, Shizuoka General Hospital, Shizuoka and ${ }^{4}$ Department of Clinical Pathology and Laboratory Medicine, Kawasaki Medical School, Kurashiki

Received for publication October 14, 2005; Accepted for publication February 15, 2006

Correspondence to Mitsuru Itoh, Department of Clinical Pathology and Laboratory Medicine, Kawasaki Medical School, 577 Matsushima, Kurashiki 701-0192
} 
administration of nitroglycerin. An electrocardiogram revealed ST elevation in leads $\mathrm{I}$ and $\mathrm{aV}_{\mathrm{L}}$, and his serum creatine phosphokinase level rose to 256 IU/l. Emergency coronary angiography showed total occlusion of the left anterior descending branch (first diagonal branch: \#9), and myocardial infarction was diagnosed. Percutaneous transluminal coronary angioplasty (PTCA) was attempted, but it was unsuccessful because the coronary embolization was too solid. The condition of the patient was stable after PTCA was attempted. On May 10, 2000, he suddenly went into cardiac and respiratory arrest. Cardiopulmonary resuscitation was attempted, but he died.

An autopsy revealed the presence of disseminated aspergillosis, involving the lungs, heart and brain. Microscopic examination showed Grocott-Gomori methenamine-silver nitrate stain positive and dichotomously branching hyphae of Aspergillus (Fig. 1). The largest Aspergillus infection focus, with a diameter of seven centimeters, was present in the superior lobe of the left lung. Severe left pulmonary infarction due to direct arteriovenous fungal invasion and disseminated bilateral fungal pneumonia extending to the pleura, pericardium and ribs were found. Myocardial infarction was observed. The diagonal branch of the left coronary artery was occluded by hematogenous Aspergillus embolizations, and a transmural infarction was present in the anterior wall of the left ventricle. Furthermore, pulmonary aspergillosis had directly invaded the epicardium and myocardium. Widespread fibrinous pyopericarditis was observed, and extensive multifocal areas of myocardial necrosis due to fungal plugs were also present. The hyphae had reached the intracardiac lumen. In addition, fungal vegetation was found on the occipital lobe of the left brain.

\section{Discussion}

Various infections become complicated by severe immunosuppression due to hematological disorders and immunosuppressive therapies. Disseminated aspergillosis occurs in immunocompromised hosts. In particular, cardiac aspergillosis is one of the most serious complications of systemic aspergillosis. Cardiac aspergillosis has been encountered as a complication in patients after cardiovascular surgery, but it is rare in patients with no history of cardiac surgery (7). It is divided broadly into two categories: direct Aspergillus invasion and Aspergillus embolization. Various clinical symptoms have been reported, including myocardial infarction (1-6), myocarditis (6-10), endocarditis (7, 18-22), pericarditis $(7,8,10-17)$, pericardial tamponade $(11,13,16)$, superior vena cava syndrome (23), aortitis (24), intracardiac mass (25) and atrioventricular block (9).

Myocardial infarction due to Aspergillus embolization often becomes a problem in the differential diagnosis with common myocardial infarction. For hemodynamic reasons, an embolic event is likely to engage the descending branch of the left coronary artery (1). Generally speaking, in these cases, it is very difficult to diagnose these symptoms as As-
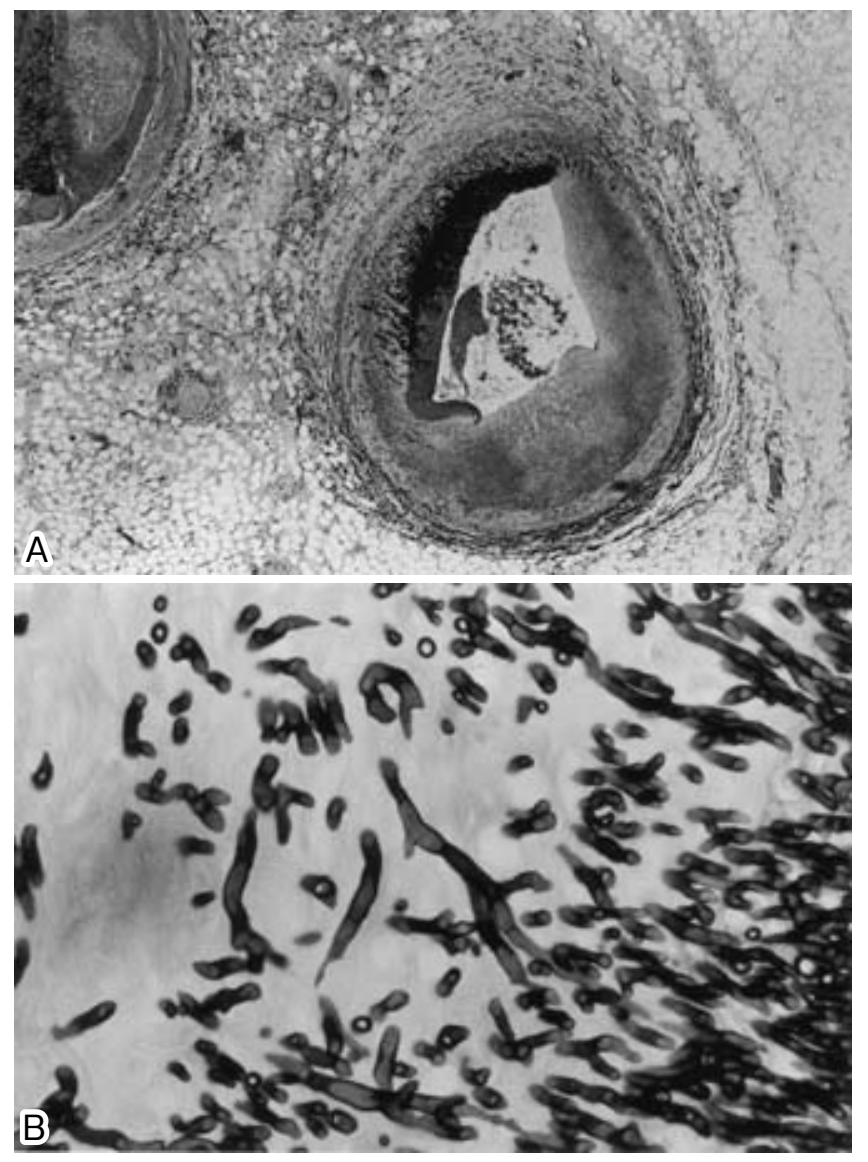

Figure 1. (A) Cross-section of the coronary artery of a patient with a thrombus occluding the artery (Grocott-Gomori methenamine-silver nitrate staining; $\times 40$ ). (B) High-power views of the section from (A), showing invasion of aspergillus (Grocott-Gomori methenamine-silver nitrate staining; $\times 400$ ).

pergillus (26). Furthermore, treatment for cardiac aspergillosis is very difficult, even when accurately diagnosed. Cardiac aspergillosis is rarely diagnosed before death, and it is fatal. Amphotericin B (with or without 5-flucytosine), itraconazole and surgical procedures are chosen for treatment $(6,11,12,16,27)$. Treatment has been successful in a few cases $(12,16,28)$, but most patients have died. Cultures of various specimens, chest X-rays and serological assays are used for diagnosis, but they are often inferior in sensitivity and specificity (29). Some reports suggest that the polymerase chain reaction assay $(22,30,31)$ and the enzymelinked immunosorbent assay (32) are useful for diagnosis. Walsh et al reported that pulmonary aspergillosis preceded development of pericarditis (13). In the present case, the patient had been neutropenic for a few months prior to the onset of cardiac problems, but he had no cardiac problems before this episode. He had no history of cardiac surgery, and he had few risk factors (smoking, hypertension, diabetes, hyperlipidemia, familiar history, and so on) of myocardial infarction except for slight obesity. We made a diagnosis of Aspergillus infection before his death based on a pneumonia image on a chest X-ray and a positive serum Aspergillus an- 
tigen finding. Thereafter we administered amphotericin B. We had wanted to administer a larger quantity (1.0-1.5 mg/ $\mathrm{kg} /$ day) of amphotericin B to the patient, but we were unable to because he had only one kidney and his renal function was not adequate. Therefore we used an insufficient quantity $(0.5-0.7 \mathrm{mg} / \mathrm{kg} /$ day) of amphotericin B. We could not use other antifungal drugs because of drug eruption. Unfortunately, the onset of myocarditis and myocardial infarction could not be prevented. Supplementarily, we tried cultures of sputum, blood and autopsy specimens, but we could not identify which Aspergillus species was involved. Aspergillus fumigatus antigen was negative.

The spread of the Aspergillus infection to the patient's heart appears to have been by both hematogenous spread from the involved pulmonary veins and direct invasion from pulmonary aspergillosis. There have been several published cases of cardiac aspergillosis, and myocardial infarction caused by Aspergillus embolization to the coronary arteries has been reported by several authors. In the present case, we considered that the patient died of myocardial infarction as well. In addition, he had complicated myocarditis due to direct infiltration from Aspergillus pneumonia to the cardiac muscle. Generally, when patients with a severe immunosup- pressed condition, such as in the present case, have an Aspergillus infection, it may be difficult to cure aspergillosis even if it is treated aggressively $(10,11)$. However, empirical use of amphotericin B should be initiated if the immunocompromised patient has a persistent fever and antibiotics have been ineffective (33). Prevention of fungal infection from the early stage, early diagnosis using various diagnostic procedures and early initiation of therapy are very important (1). Recently, new antifungal agents for the treatment of aspergillosis have become available: caspofungin (34), micafungin (35) and voriconazole (36). These drugs have potent antifungal action including action against Aspergillus. Herbrecht et al reported that voriconazole is more effective and has fewer side effects than amphotericin B in patients with invasive aspergillosis (36). Maertens et al showed that caspofungin is useful in the salvage treatment of invasive aspergillosis (34). These drugs are expected as a new treatment strategy for aspergillosis.

It is difficult to treat systemic aspergillosis, but we should attempt to detect the symptoms of pulmonary aspegillosis, pericarditis and myocardial infarction as soon as possible, and cardiac aspergillosis should be suspected in such a situation.

\section{References}

1. Andersson BS, Luna MA, McCredie KB. Systemic aspergillosis as cause of myocardial infarction. Cancer 58: 2146-2150, 1986.

2. Laszewski M, Trigg M, de Alarcon P, Giller R. Aspergillus coronary embolization causing acute myocardial infarction. Bone Marrow Transplant 3: 229-233, 1988.

3. Cishek MB, Yost B, Schaefer S. Cardiac aspergillosis presenting as myocardial infarction. Clin Cardiol 19: 824-827, 1996.

4. Ross EM, Macher AM, Roberts WC. Aspergillus fumigatus thrombi causing total occlusion of both coronary arterial ostia, all four major epicardial coronary arteries and coronary sinus and associated with purulent pericarditis. Am J Cardiol 56: 499-500, 1985.

5. Landonio G, Nosari A, Gargantini L, De Cataldo F, Oreste P. Pulmonary and myocardial infarction secondary to arterial occlusion by Aspergillus fumigatus in ANLL. Haematologica 74: 503-505, 1989.

6. Kuijer PM, Kuijper EJ, van den Tweel JG, van der Lelie J. Aspergillus fumigatus, a rare cause of fatal coronary artery occlusion. Infection 20: 45-47, 1992.

7. Sergi C, Weitz J, Hofmann WJ, et al. Aspergillus endocarditis, myocarditis and pericarditis complicating necrotizing fasciitis. Case report and subject review. Virchows Arch 429: 177-180, 1996.

8. Schwartz DA. Aspergillus pancarditis following bone marrow transplantation for chronic myelogenous leukemia. Chest 95: 1338-1339, 1989.

9. Rogers JG, Windle JR, McManus BM, Easley AR Jr. Aspergillus myocarditis presenting as myocardial infarction with complete heart block. Am Heart J 120: 430-432, 1990.

10. Ozsahin H, Wacker P, Brundler MA, et al. Fatal myocardial aspergillosis in an immunosuppressed child. J Pediatr Hematol Oncol 23: 456-459, 2001.

11. Luce JM, Ostenson RC, Springmeyer SC, Hudson LD. Invasive aspergillosis presenting as pericarditis and cardiac tamponade. Chest 76: 703-705, 1979.
12. Cooper JA, Weinbaum DL, Aldrich TK, Mandell GL. Invasive aspergillosis of the lung and pericardium in a nonimmunocompromised 33 year old man. Am J Med 71: 903-907, 1981.

13. Walsh TJ, Bulkley BH. Aspergillus pericarditis: clinical and pathologic features in the immunocompromised patient. Cancer 49: 48-54, 1982.

14. Carrel TP, Schaffner A, Schmid ER, et al. Fatal fungal pericarditis after cardiac surgery and immunosuppression. J Thorac Cardiovasc Surg 101: 161-164, 1991.

15. van Ede AE, Meis JF, Koot RA, Heystraten FM, de Pauw BE. Pneumopericardium complicating invasive pulmonary aspergillosis: case report and review. Infection 22: 102-105, 1994.

16. Le Moing V, Lortholary O, Timsit JF, et al. Aspergillus pericarditis with tamponade: report of a successfully treated case and review. Clin Infect Dis 26: 451-460, 1998.

17. Gokahmetoglu S, Koc AN, Patiroglu T. Case report. Fatal Aspergillus flavus pericarditis in a patient with acute myeloblastic leukaemia. Mycoses 43: 65-66, 2000.

18. Walsh TJ, Hutchins GM. Aspergillus mural endocarditis. Am J Clin Pathol 71: 640-644, 1979.

19. Freischlag JA, Asbun HA, Sedwitz MM, et al. Septic peripheral embolization from bacterial and fungal endocarditis. Ann Vasc Surg 3: 318-323, 1989.

20. Schett G, Casati B, Willinger B, et al. Endocarditis and aortal embolization caused by Aspergillus terreus in a patient with acute lymphoblastic leukemia in remission: diagnosis by peripheralblood culture. J Clin Microbiol 36: 3347-3351, 1998.

21. Viertel A, Ditting T, Pistorius K, et al. An unusual case of Aspergillus endocarditis in a kidney transplant recipient. Transplantation 68: 1812-1813, 1999.

22. McCracken D, Barnes R, Poynton C, et al. Polymerase chain reaction aids in the diagnosis of an unusual case of Aspergillus niger endocarditis in a patient with acute myeloid leukaemia. J Infect 47: 344-347, 2003.

23. Takatsuka H, Wakae T, Mori A, et al. Superior vena cava syn- 
drome after bone marrow transplantation caused by aspergillosis: a case report. Hematology 7: 169-172, 2002.

24. Sanchez-Recalde A, Mate I, Merino JL, Simon RS, Sobrino JA Aspergillus aortitis after cardiac surgery. J Am Coll Cardiol 41: 152-156, 2003.

25. Berarducci L, Ford K, Olenick S, Devries S. Invasive intracardiac aspergillosis with widespread embolization. J Am Soc Echocardiogr 6: 539-542, 1993.

26. Atkinson JB, Connor DH, Robinowitz M, McAllister HA, Virmani R. Cardiac fungal infections: review of autopsy findings in $60 \mathrm{pa}-$ tients. Hum Pathol 15: 935-942, 1984.

27. Ramos AdeO, Medeiros AR, Paulista PP, Abboud CS, Meneghelo $\mathrm{ZM}$. Aspergillus infection in the ascending aorta of a patient with aortic and mitral valve prostheses. Arq Bras Cardiol 81: 419-420, 2003 (in English/Portuguese).

28. Swensson EE, Willman VL, Peterson GJ. Acute aortic occlusion from aspergillosis in a healthy patient with survival. J Vasc Surg 4: 187-191, 1986.

29. Vo NM, Russell JC, Becker DR. Mycotic emboli of the peripheral vessels: analysis of forty-four cases. Surgery 90: 541-545, 1981.

30. Kanda Y, Akiyama H, Onozawa Y, et al. Aspergillus endocarditis in a leukemia patient diagnosed by a PCR assay. Kansenshogaku Zasshi 71: 269-272, 1997.

31. Kami M, Fukui T, Ogawa S, et al. Use of real-time PCR on blood samples for diagnosis of invasive aspergillosis. Clin Infect Dis 33: 1504-1512, 2001.

32. Machetti M, Feasi M, Mordini N, et al. Comparison of an enzyme immunoassay and a latex agglutination system for the diagnosis of invasive aspergillosis in bone marrow transplant recipients. Bone Marrow Transplant 21: 917-921, 1998.

33. Pizzo PA, Robichaud KJ, Gill FA, Witebsky FG. Empiric antibiotic and antifungal therapy for cancer patients with prolonged fever and granulocytopenia. Am J Med 72: 101-111, 1982.

34. Maertens J, Raad I, Petrikkos G, et al. Efficacy and safety of caspofungin for treatment of invasive aspergillosis in patients refractory to or intolerant of conventional antifungal therapy. Clin Infect Dis 39: 1563-1571, 2004.

35. Carver PL. Micafungin. Ann Pharmacother 38: 1707-1721, 2004.

36. Herbrecht R, Denning DW, Patterson TF, et al. Voriconazole versus amphotericin B for primary therapy of invasive aspergillosis. N Engl J Med 347: 408-415, 2002.

(C) 2006 The Japanese Society of Internal Medicine http://www.naika.or.jp/imindex.html 